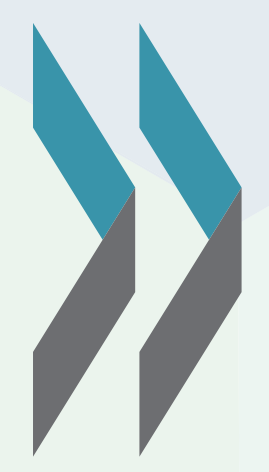

\title{
L'excès de tests est-il préjudiciable à la performance et au bien-être des élèves?
}

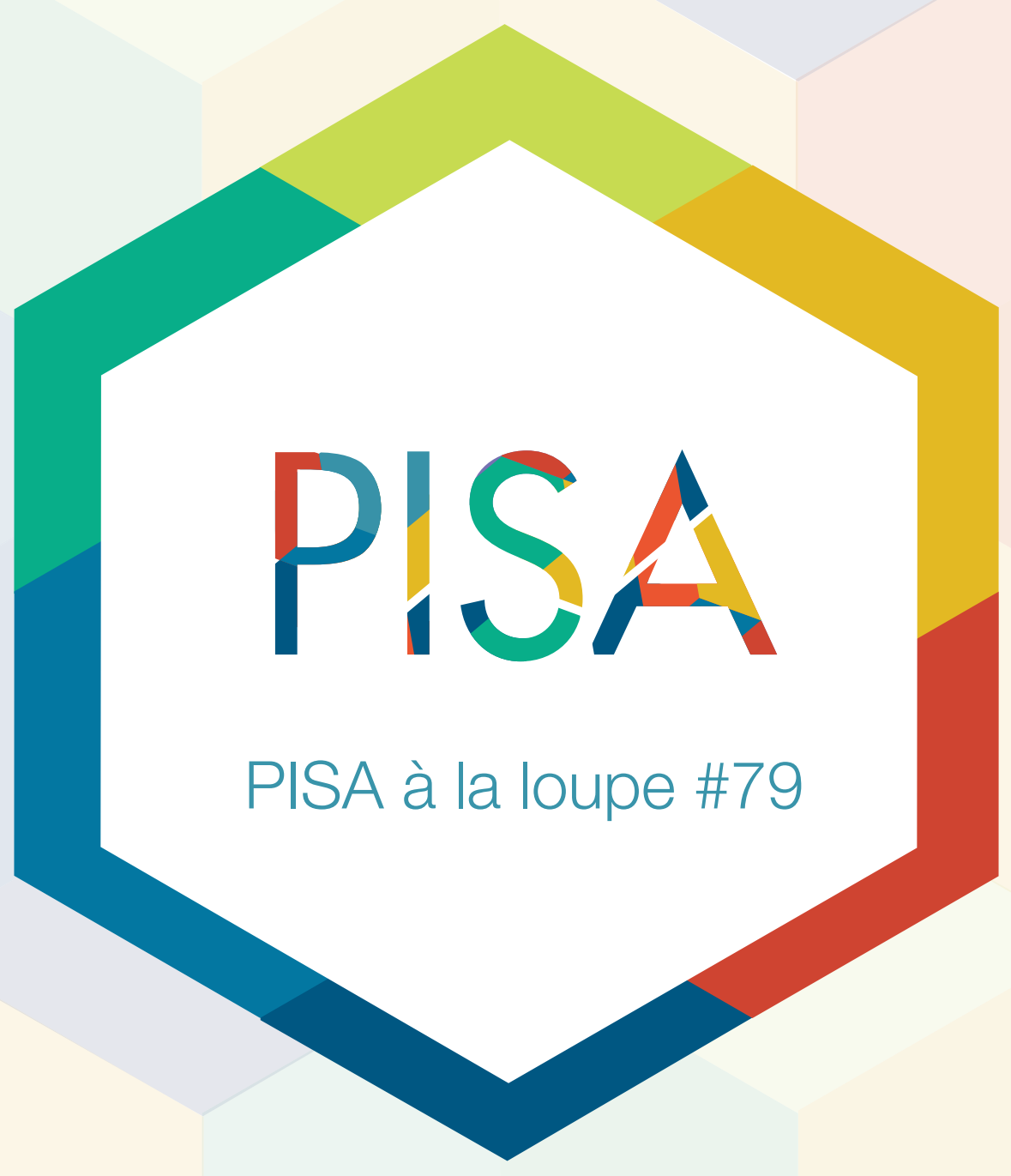




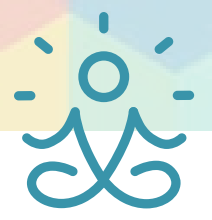

\section{L'excès de tests est-il préjudiciable à la performance et au bien-être des élèves?}

- En moyenne, 70 \% des élèves fréquentent des établissements qui n'administrent jamais ou qu'une ou deux fois par an de tests standardisés. Parallèlement, plus de $60 \%$ des élèves sont évalués au moins une fois par mois à l'aide d'épreuves mises au point par les enseignants et d'appréciations informelles fournies par ces derniers.

- La prévalence de l'anxiété liée au travail scolaire est forte : $59 \%$ des élèves ont peur de ne pas réussir leurs contrôles et 66 \%, d'avoir de mauvaises notes.

- Aucune relation ne s'observe entre d'une part, la fréquence des tests et, d'autre part, l'anxiété vis-à-vis des tests et la performance en sciences.

Les tests standardisés aident à évaluer les progrès des élèves à l'école et permettent d'éclairer les politiques d'éducation sur les lacunes existantes. Toutefois, l'excès de tests pourrait faire peser une forte pression sur les élèves et les enseignants si l'apprentissage et l'enseignement sont orientés en fonction des épreuves, ce qui nuirait au final au plaisir d'apprendre.

Nombre de parents et de professionnels de l'éducation ne connaissent que trop bien ce dilemme : tout en reconnaissant le potentiel d'amélioration inhérent aux bonnes évaluations, ils s'inquiètent souvent du fait que l'excès de tests puisse être anxiogène pour les élèves, sans pour autant les faire progresser dans leurs apprentissages. Les tests standardisés décisifs pour le parcours scolaire et personnel des élèves peuvent en particulier être sources d'anxiété et, s'ils sont administrés trop fréquemment, entraîner une diminution de la performance, une hausse de l'absentéisme et une baisse de la confiance en soi. Mais la prévalence des tests standardisés est-elle réellement si forte ? Et que nous apprennent les données sur la relation entre performance, anxiété et fréquence des tests ?

\section{Contrairement aux idées reçues, la prévalence des tests standardisés n'est pas si forte.}

Dans le cadre de l'enquête PISA 2015, des données ont été collectées sur la fréquence des tests au cours de l'année d'études généralement fréquentée par les jeunes de 15 ans, ainsi que sur le niveau auto-déclaré d'anxiété des élèves. II en ressort que les tests standardisés - obligatoires ou non - ne sont pas utilisés aussi fréquemment que les épreuves mises au point par les enseignants. En moyenne, dans les pays de l'OCDE, quelque $25 \%$ des élèves de 15 ans fréquentent un établissement qui n'administre jamais de tests standardisés obligatoires, et $60 \%$, un établissement qui n'administre ce type de tests qu'une ou deux fois par an. Dans 11 pays, notamment en Allemagne, en Belgique, au Costa Rica, en Espagne et en Slovénie, plus de 50 $\%$ des élèves fréquentent des établissements qui n'utilisent jamais de tests standardisés obligatoires pour les évaluer, tandis qu'au Royaume-Uni et en Suède, tous les élèves passent ce type de tests au moins une fois durant l'année scolaire considérée (par exemple, le GCSE en Angleterre). Si les tests non obligatoires sont administrés moins fréquemment que les tests obligatoires, l'utilisation des épreuves mises au point par les enseignants et des appréciations informelles de ces derniers est considérablement plus forte. En moyenne, dans les pays de l'OCDE, quelque $30 \%$ des élèves passent chaque mois des épreuves mises au point par leurs enseignants et $38 \%$, plus d'une fois par mois (une proportion qui s'élève à plus de $50 \%$ des élèves en Belgique, au Canada, en Espagne, en France, aux Pays-Bas, à Singapour et au Taipei chinois).

II est intéressant de noter qu'aux États-Unis, qui ont vu naître le débat sur les tests standardisés, moins de 30 \% des élèves fréquentent des établissements qui administrent des tests obligatoires au moins trois fois par an - soit une proportion inférieure à celle observée dans 19 autres systèmes d'éducation.

Les données de l'enquête PISA révèlent en outre la forte prévalence de l'anxiété liée aux tests. En moyenne, quelque 59 \% des élèves disent avoir peur d'avoir des difficultés à réussir un contrôle, 66 \%, avoir peur d'avoir des mauvaises notes, 55 \%, se sentir très angoissés même lorsqu'ils se sont bien préparés pour un contrôle, 52 \%, devenir nerveux quand ils ne savent pas comment résoudre un exercice à l'école, et 37 \% être très tendus quand ils étudient pour un contrôle. 
Fréquence des évaluations à l'école

Pourcentage d'élèves fréquentant des établissements où les pratiques d'évaluation suivantes sont utilisées ; moyenne OCDE

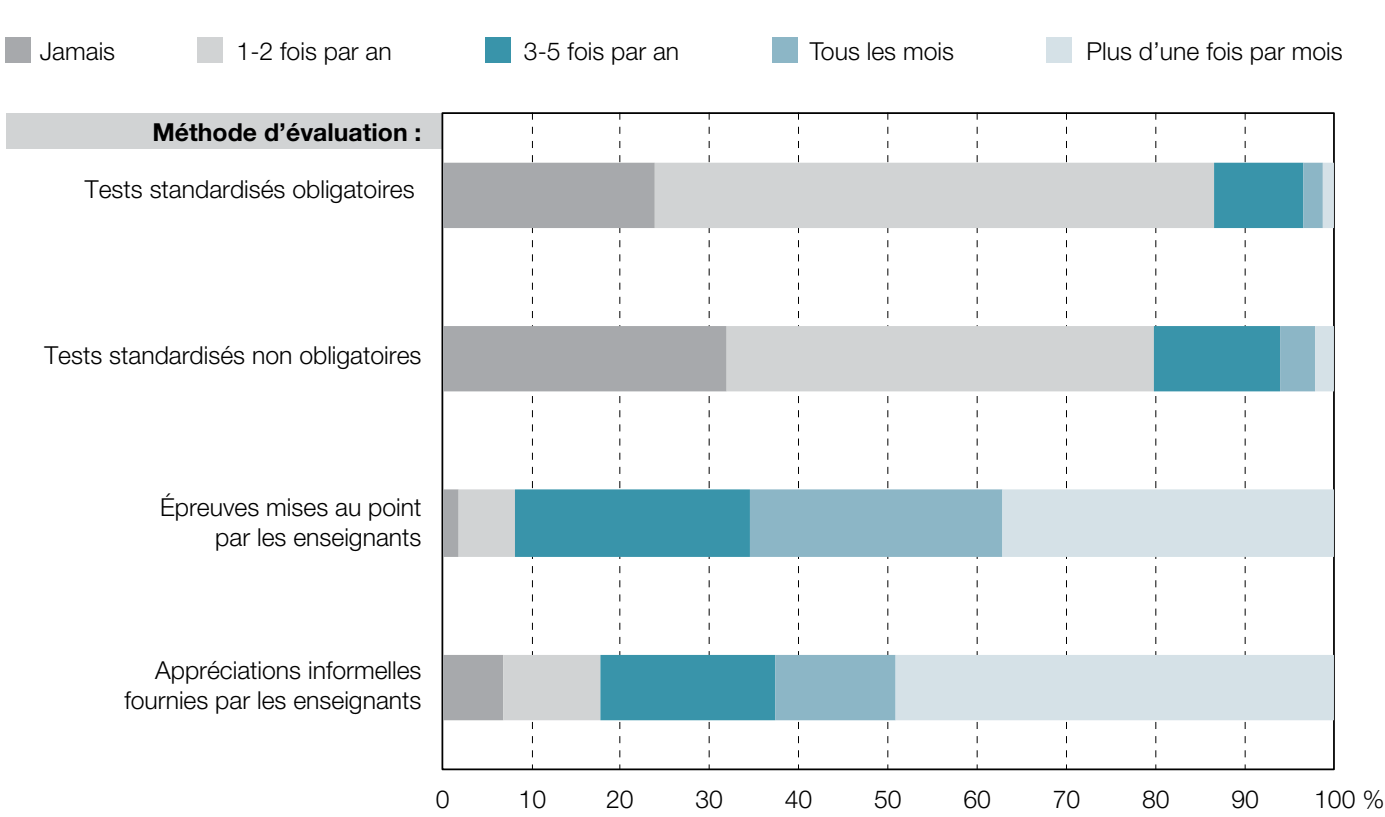

Source: OCDE, Base de données PISA 2015, tableau II.4.19.

StatLink Ails http://dx.doi.org/10.1787/888933435972

Prévalence de l'anxiété liée au travail scolaire, selon le sexe

Pourcentage d'élèves se disant « d'accord » ou " tout à fait d'accord » avec les affirmations suivantes ; moyenne OCDE

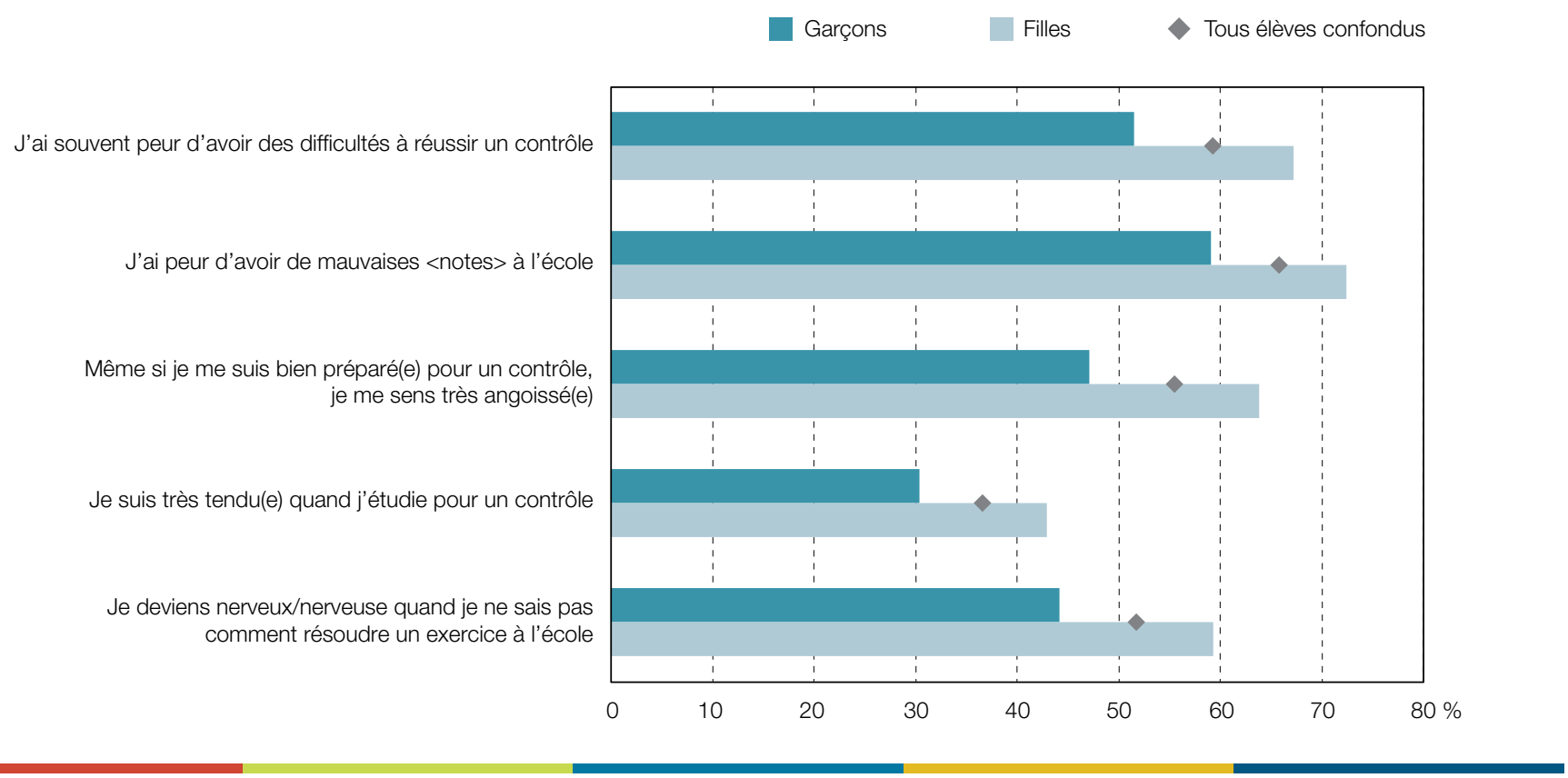

Remarque : Toutes les différences entre les sexes sont statistiquement significatives (voir l'annexe A3 dans OCDE [2016], Résultats du PISA 2015 Nolume II], Éditions OCDE, Paris).

Source : OCDE, Base de données PISA 2015, tableaux III.4.1, III.4.2 et III.4.5

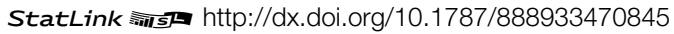




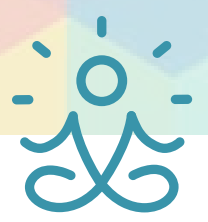

\section{L'anxiété des élèves vis-à-vis des tests n'a pas grand-chose à voir avec la fréquence de ces derniers.}

Contrairement aux idées reçues, aucun lien ne s'observe entre la fréquence des tests, telle que communiquée par les chefs d'établissement, et le niveau d'anxiété vis-à-vis des tests dont font part les élèves. De fait, en moyenne, dans les pays de l'OCDE, les élèves qui fréquentent des établissements où ils doivent passer au moins une fois par mois des tests standardisés ou des épreuves mises au point par leurs enseignants font part de niveaux d'anxiété vis-à-vis des tests similaires à ceux observés chez les élèves fréquentant des établissements où l'utilisation de ce type de tests est moins fréquente.

Parmi les explications possibles, l'une pourrait tenir au fait que l'anxiété vis-à-vis des tests dépendrait d'autres caractéristiques que leur fréquence. La nature ou la difficulté de la tâche, l'atmosphère environnante, les contraintes de temps, les caractéristiques de l'examinateur, le mode d'administration et l'environnement physique du test peuvent par exemple influer sur les attitudes psychologiques des élèves vis-à-vis de l'épreuve, autant de facteurs qui interagissent à leur tour avec les capacités de l'élève, sa confiance en soi, sa motivation, ses compétences d'apprentissage et de réponse au test, et sa préparation.

La relation entre la performance en sciences et la fréquence à laquelle les établissements ou les pays évaluent les élèves est également faible. En moyenne, dans les pays de l'OCDE, les élèves évalués à l'aide de tests standardisés obligatoires au moins une fois par an obtiennent un score légèrement inférieur en sciences (de 6 points) à celui de leurs pairs évalués plus fréquemment, tandis que les élèves évalués au moins une fois par mois à l'aide d'épreuves mises au point par leurs enseignants obtiennent un score légèrement supérieur (de 5 points) à celui de leurs pairs évalués moins fréquemment. Toutefois, après contrôle du profil socio-économique des élèves et des établissements, ces associations ne sont pas significatives.

La performance d'un pays aux épreuves PISA semble également sans lien avec l'utilisation de tests standardisés. Parmi les pays et économies dans le haut du classement en sciences, l'utilisation de tests standardisés obligatoires est ainsi répandue dans l'entité Beijing-Shanghai-Jiangsu-Guangdong (Chine), au Royaume-Uni et à Singapour, mais relativement peu courante en Allemagne, en Corée et en Suisse.

\section{Méthodes d'évaluation à l'école et performance en sciences \\ Corrélations au niveau Système}

\begin{tabular}{l|c|c}
\multirow{2}{*}{\begin{tabular}{l}
\multicolumn{1}{c|}{ Méthodes d'évaluation à l'école et performance en sciences } \\
\cline { 2 - 3 }
\end{tabular}} & Dans les pays de l'OCDE & Dans 64 pays et économies \\
\cline { 2 - 3 } Tests standardisés obligatoires au moins une fois par an & Performance en sciences & 0.05 \\
\hline Tests standardisés non obligatoires au moins une fois par an & -0.04 & 0.12 \\
\hline $\begin{array}{l}\text { Épreuves mises au point par les enseignants au moins une fois par } \\
\text { mois }\end{array}$ & 0.15 & 0.15 \\
\hline
\end{tabular}

Remarque : Le coefficient de corrélation varie de -1 à 1 et indique l'existence d'une association linéaire entre les deux variables. Les valeurs proches de 0 indiquent l'absence d'association linéaire; celles proches de 1, l'existence d'une forte relation positive ; et celles proches de -1, l'existence d'une forte relation négative. Les valeurs statistiquement significatives sont indiquées en gras (voir l'annexe A3 dans OCDE [2017], Résultats du PISA 2015 Nolume II], Éditions OCDE, Paris).

Source : OCDE, Base de données PISA 2015.

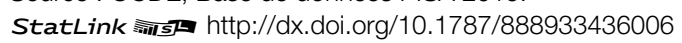

\section{L'anxiété des élèves vis-à-vis des tests est moins forte lorsqu'ils se sentent soutenus par leurs enseignants et leurs parents.}

Dans l'expérience scolaire des élèves, d'autres aspects que la fréquence des évaluations sont en plus forte relation avec leur probabilité de souffrir d'anxiété. D'après l'enquête PISA, les élèves font part d'un niveau plus faible d'anxiété lorsque les enseignants leur apportent davantage de soutien ou adaptent leurs cours à leurs besoins. En revanche, ils font part d'une plus grande anxiété quand ils ont le sentiment que les enseignants les traitent de façon injuste, par exemple lorsqu'ils les notent plus sévèrement que les autres élèves ou qu'ils pensent qu'ils sont moins intelligents qu'ils ne le sont vraiment. 


\section{Pratiques des enseignants et anxiété liée au travail scolaire chez les élèves}

Probabilité pour les élèves de se sentir très angoissés même s'ils sont bien préparés pour un contrôle ou de se sentir très tendus quand ils étudient pour un contrôle, selon les pratiques des enseignants
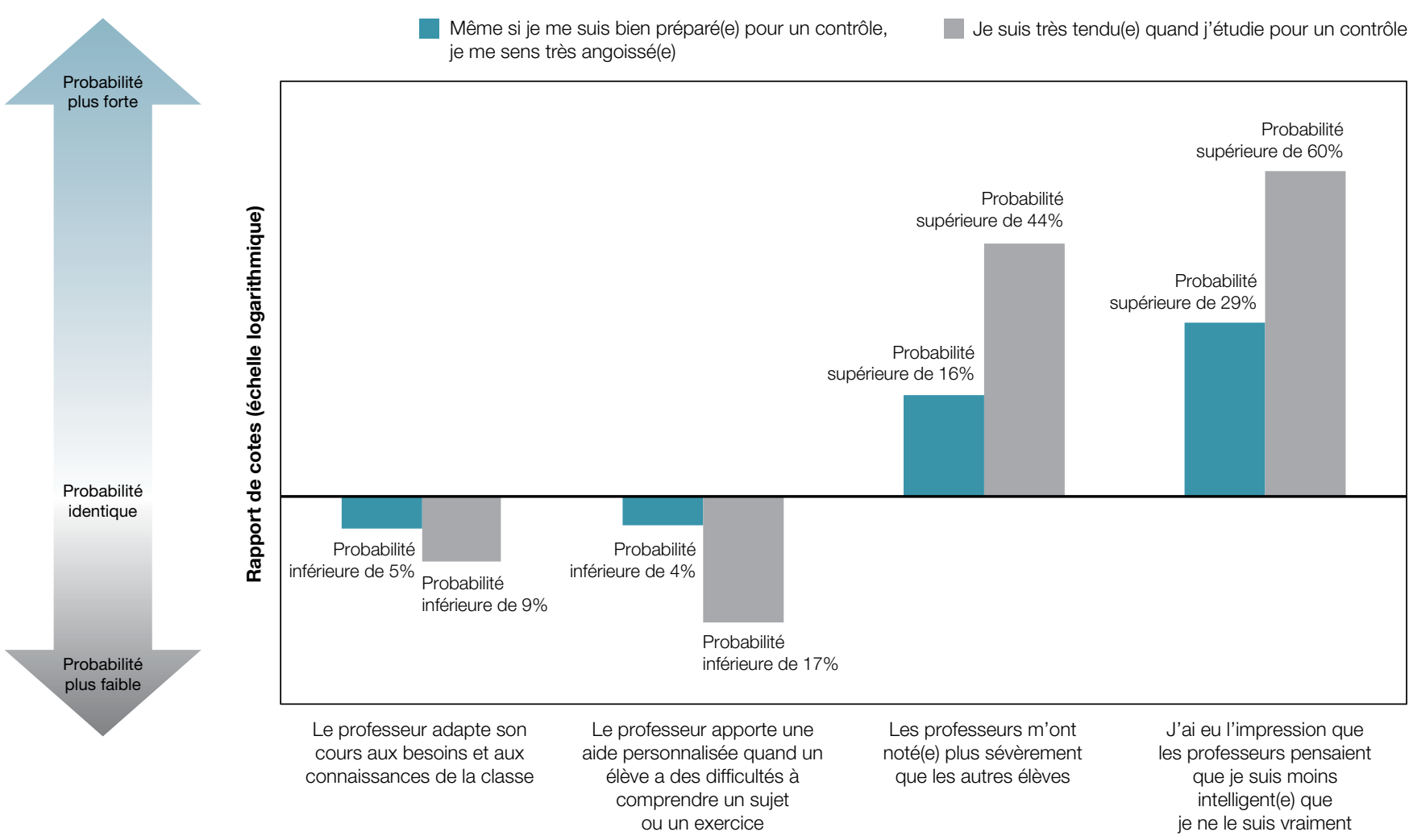

Remarque : Les valeurs tiennent compte des différences d'indice PISA de statut économique, social et culturel (SESC) et de performance en sciences entre les élèves. Toutes les valeurs sont statistiquement significatives.

Source : OCDE, Base de données PISA 2015, tableau III.4.11.

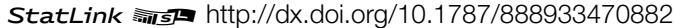

Le maintien de relations positives avec les parents est également essentiel. Ils peuvent en effet aider leurs enfants à surmonter leur anxiété en les encourageant à croire en leurs capacités à réussir ce qu'on leur demande à l'école. Ainsi, les filles déclarant que leurs parents les encouragent à avoir confiance en elles font part d'un niveau d'anxiété largement inférieur, même après contrôle des différences de performance et de statut socio-économique (cette relation s'avère plus faible chez les garçons). À l'inverse, le fait d'accorder trop d'importance aux résultats des tests et de fixer des objectifs irréalistes ne fait qu'accroître l'anxiété.

\section{Pour conclure}

Les tests standardisés et ceux mis au point par les enseignants jouent un rôle important dans le suivi de la performance des élèves et des progrès scolaires. Les élèves qui ont le sentiment que leurs enseignants les traitent équitablement et les aident, à l'instar de leurs parents, à renforcer leur confiance en eux tout en fixant des objectifs réalistes, sont moins susceptibles de se sentir anxieux face aux tests. 


\section{Pour tout complément d'information}

Contacter : Tarek Mostafa (Tarek.Mostafa@oecd.org)

Consulter : OCDE (2017), Résultats du PISA 2015 (Volume II) : Politiques et pratiques pour des établissements performants, PISA, Éditions OCDE, Paris, http://dx.doi.org/10.1787/9789264267558-fr.

OCDE (2017), Résultats du PISA 2015 Nolume III) : Le bien-être des élèves, PISA, Éditions OCDE, Paris, http://dx.doi.org/10.1787/9789264288850-fr.

Le mois prochain : Dans quels pays et établissements les élèves défavorisés réussissent-ils ?

Cet ouvrage est publié sous la responsabilité du Secrétaire général de l'OCDE. Les opinions et les interprétations exprimées ne reflètent pas nécessairement les avis des pays membres de l'OCDE.

Ce document, ainsi que les données et cartes qu'il peut comprendre, sont sans préjudice du statut de tout territoire, de la souveraineté s'exerçant sur ce dernier, du tracé des frontières et limites internationales, et du nom de tout territoire, ville ou région.

Ce texte est disponible sous licence Attribution - Pas d'Utilisation Commerciale - Partage dans les Mêmes Conditions 3.0 Organisations Internationales

(CC BY-NC-SA 3.0 IGO). Pour toute information spécifique quant à l'étendue et aux termes de la licence ainsi que d'une possible utilisation commercial de ce texte et pour toute usage de données PISA, prière de consulter les Conditions d'utilisation à http://www.oecd.org/fr/conditionsdutilisation. 